\title{
The Relationship between Signs and Symptoms of Tempromandibular Joint Disorders (TMDs) and Primary Headache \\ Zena Kamel kadhem
}

B.D.S,Msc. Oral Medicine.

Muntaha Fawzi

B.D.S,Msc. Oral Medicine

Sabah Farhan

B.D.S, Msc. Oral Medicine

\section{ABSTRACT}

The aims of this study are to investigate the relationship of the concurrent signs and symptoms of a painful TMD with primary headache (migraine and tension type headache) and whether tenderness and provocation of the masticatory system resulted in the headache.

Methods and materials: The sample consists of 120 patients which were diagnosed as having temporomandibular joint disorder (TMD) included Research Diagnostic Criteria (RDC). The sample age range was between (18-50 years). All patients oriented to complete the Research Diagnostic Criteria RDC ITMD. The questionnaire for TMD index was also applied to all patients to measure the severity of TMD. Then all patients were subjected to clinical examination (examination of masticatory muscles, joint palpation and deviation, clicking and limitation in mouth opening). Primary Headaches were assessed using a validated structured questionnaire captured information on distinguishing headache features and parallels the International Classification for Headache Disorders-II. Results: females were shown high prevalence of TMDs and headache more than male. The presence of the masticatory muscles pain, joint pain, limitation in mouth opening, clicking and bruxism as well as headache pain intensity and duration, increased to a relevant and statistically significant degree in both migraine and tension type headache patients in compare to other patients had no headache or patients with headache from TMDs.

Conclusions: High prevalence of TMD in females and also females represent a majority of headache patients. Clinical pain characteristics and masticatory muscle tenderness of TMD are greater in patients with primary headache.

\section{KEYWORDS}

TMDs, Tension-type headache, Migraine, RDC\TMD

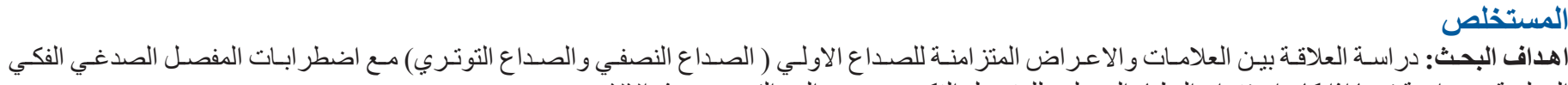

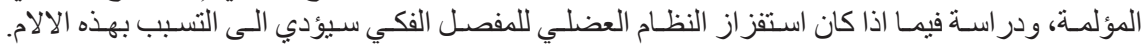

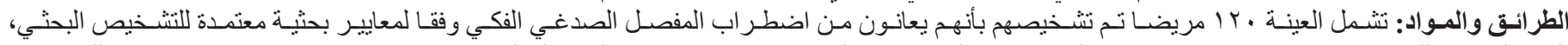

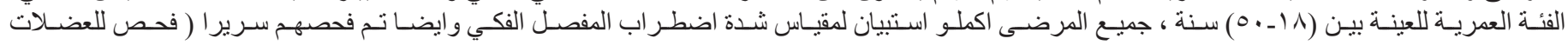

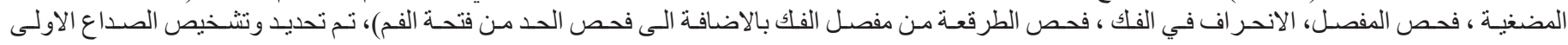

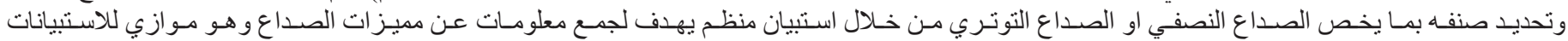

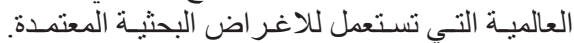

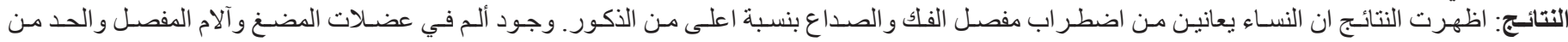

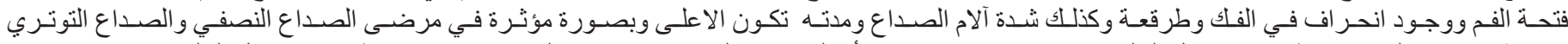

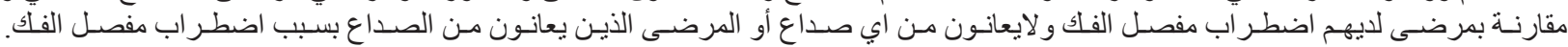

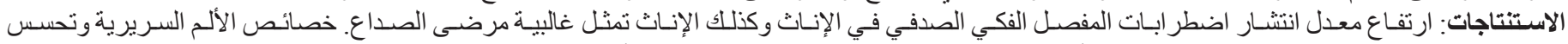

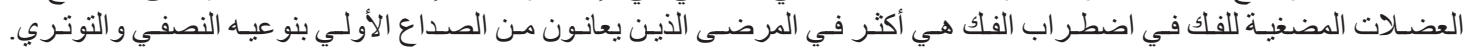

\section{INTRODUCTION}

The temporomandibular joint (TMJ) is a synovial articulation between the mandibular condyle and the squamous portion of the temporal bone. The TMJ is one of the most complex, delicate and highly used joint in human body ${ }^{(1)}$. Several conditions may affect the TMJ called Tempromandibular disorders, TMD is a collective term, embracing several clinical problems involving the muscles, temporomandibular joint (TMJ), or both ${ }^{(2)}$. Tempromandibular disorder is a symptom complex rather than a single condition, and it is thought to be caused by multiple factors like: genetic, developmental, and metabolic ${ }^{(3)}$. TMDs form a cluster of related disorders with common symptoms which include localized pain, limited or asymmetric movement, and clicks or grating on opening ${ }^{(4)}$.Signs and symptoms of temporomandibular joint disorder vary in their presentation. The symptoms will usually involve more than one of the various components of the masticatory system, muscles, nerves, tendons, ligaments, bones, connective tissue, and/or the teeth (4).

The three classically described, cardinal signs and symptoms of TMD are:

- Pain and tenderness on palpation of the muscles of mastication, or of the joint itself (preauricular pain - pain felt just in front of the ear). Pain is the defining feature of TMD and is usually aggravated by manipulation or function, such as when chewing, clenching, or yawning ${ }^{(5)}$. The character of the pain is usually dull or aching, poorly localized, and intermittent, although it can sometimes be constant. The pain is more usually unilateral (located on one side) rather than bilateral. It is rarely severe ${ }^{(6)}$.

- Limited range of mandibular movement, which may cause difficulty in eating or even talking. 
There may be locking of the jaw, or stiffness in the jaw muscles and the joints. There may also be incoordination, asymmetry or deviation of mandibular movement ${ }^{(7)}$.

- Noises from the joint during mandibular movement, which may be intermittent. Joint noises may be described as clicking, popping, or crepitus ${ }^{(8)}$.

The International Headache Society's diagnostic criteria for "headache or facial pain attributed to temporomandibular joint disorder" ${ }^{(9)}$ is similar to the:

a. Recurrent pain in one or more regions of the head and/or face fulfilling criteria $\mathrm{C}$ and $\mathrm{D}$

b.X-ray, MRI and/or bone scintigraphy demonstrate TMJ disorder

c.Evidence that pain can be attributed to the TMJ disorder, based on at least one of the following:

- pain is precipitated by jaw movements and/or chewing of hard or tough food

- reduced range of or irregular jaw opening

- noise from one or both TMJs during jaw movements

- tenderness of the joint capsule(s) of one or both TMJs

d. Headache resolves within 3 months, and does not recur, after successful treatment of the TMJ disorder.

Various diagnostic systems for (TMD) have been described. Some consider the Research Diagnostic Criteria method the gold standard. Abbreviated to "RDC/TMD", this was first introduced in 1992 by Dworkin and LeResche in an attempt to classify temporomandibular disorders by etiology and apply universal standards for research into TMD. This method involves 2 diagnostic axes, namely axis I, the physical diagnosis, and axis II, the psychologic diagnosis. Axis I contains 3 different groups which can occur in combinations of 2 or all 3 groups ${ }^{(10)}$. More than half of the headache patients also had TMD diagnosis according to the Research Diagnostic Criteria for Temporomandibular Disorders (RDC/TMD) ${ }^{(11)}$ and International classification of headache disorders-II (ICHD-II) ${ }^{(12)}$, indicating a close relationship, at least in some of the clinical manifestations of the painful conditions. Local pain may be viewed as the most important complaint of TMD patients. In particular, pain from the masticatory or preauricular areas is frequently reported, but also headache is one of the symptoms which often occur in TMD patients ${ }^{(12)}$.

Migraine is a familial paroxysmal neurological disorder characterized by spontaneous or triggered attacks of headache that are variably associated with autonomic disturbance like nausea or pallor, heightened sensitivity to external stimuli. The attacks usually last 4-72 hours. Migraine headaches affect $18 \%$ of women and $6 \%$ of men, although its onset is within the first three decades of life. Migraine greatly affects quality of life. The World Health Organization ranks migraine among the world's 20 most disabling medical illnesses. Family physicians and specialists in neurology seek to rule out an organic cause of headache. Unfortunately, the difficulties in distinguishing headaches based on clinical presentation rarely lead to the uncovering of an organic etiologic factor, and rarely lead to the diagnosis of comorbid conditions such as TMD. This results in frequent empiric management of headache without the evaluation of TMD as a potentially treatable comorbid condition ${ }^{(13)}$.

Due to the close anatomical relationship of the muscles of mastication and TMJ to the head and due to the frequency of referred pain, it is sensible to theorize that there is a significant percentage of patients with headache actually have TMD as the major source of their pain. The literature suggests that there is a relationship between both intracapsular articular disorders as well as disorders affecting the masticatory musculature (14,15and16). Migraine pain often manifests in areas of the head including the TMJ and masticatory muscles ${ }^{(17,18)}$. Temporomandibular disorders may cause headaches, worsen existent primary headaches, and add to the burden of headache disorders. The patients with combined migraine and tension-type headaches had a higher prevalence of temporomandibular disorders. Evidence supporting a close relationship include the increased masticatory muscle tenderness in migrainuers compared and improvement in headache symptoms with traditional TMD treatment ${ }^{(19)}$. Distinguishing between migraine and tension-type headache (TTH) can sometimes be difficult because the conditions have overlapping features, and patients have more than one type of headache. Their ability to ascribe symptoms to a specific headache on recall may be unreliable. Although TTH is the most common primary headache disorder, it is the least distinctive, most poorly understood, and most frequently mimicked by underlying diseases. In fact, its clinical diagnosis is based chiefly on the absence of the symptoms that characterize migraine ${ }^{(20)}$. The aims of this study are to investigate the relationship of the concurrent signs and symptoms of a painful TMD with primary headache (migraine and tension type headache) and whether tenderness and provocation of the masticatory system resulted in the headache 


\section{METHODS AND MATERIALS}

The sample consists of 120 patients which were diagnosed as having temporomandibular joint disorder TMD, age range between (18-50 years). The sample was collected from patients attending the oral medicine clinic at college of dentistry/ ALMustansiriya University during the period from (November 2015 to May 2016). Only one previously calibrated examiner performed all the evaluations of this research. The selected patients were interviewed by means of a questionnaire with information on their general health condition, and signs and symptoms of TMD in the last 30 days.

\section{Exclusion criteria:}

- History of arthritic disease (i.e osteoarthritis, rheumatoid arthritis).

- Patients that previously have had jaw-related trauma, surgery or TMJ injection (steroids or others in the last six months).

- Neurological disorders (Facial nerve paralysis, cerebrovascular event, trigeminal neuralgia, active psychiatric disease).

- Pregnant females.

- Patients with vision problems or sinusitis.

- Patients take medications cause headache (such as birth controls drugs) and the use of no analgesic(s) other than acetaminophen for 3 days before the exam.

All patients oriented to complete the Diagnosis Criteria for Temporomandibular disorder. McNeill $1997^{(21)}$ described TMD diagnostic criteria as follows:

- Pain in muscles of mastication, the TMJ, and /or the periauricular area (around the ear), which is usually made worse by manipulation or function.

- Asymmetric mandibular movement with or without clicking.

- Limitation of mandibular movements.

- Pain present for a minimum of 3 months.

The questionnaire for TMD index ${ }^{(22)}$ was applied to all patients with no interference by the examiner, so as not to create expectations, and leading, thus, to a possible deviation from the clinical examination to be accomplished. The patients answered ten questions related to TMD symptoms, which afforded a classification of each subject regarding the presence and severity of such dysfunctions.

\section{Anamnestic questionnaire:}

1.Do you have difficulty in opening your mouth?

2.Do you have difficulty in moving your jaw sideways?

3.Do you feel discomfort or muscular pain when chewing?
4.Do you often have headaches?

5.Do you feel pain in your neck and/or shoulders?

6.Do you feel earaches or pain near your ears?

7.Do you notice any noise in your TMJ?

8.Do you regard your bite "normal"?

9.Do you use only one side of your mouth to chew?

10.Do you feel any pain in your face when you wake up?

Three options of answers were offered for the anamnestic questionnaire: "yes", "no", or "sometimes". Value "2" was attributed to every answer indicating the presence of the symptom; value " 0 ", for the absence thereof; and value " 1 ", for the "sometimes" answer. The total sum of the values obtained allowed the classification of the sample as regards TMD, as a TMD index ${ }^{(19)}$.

- Values of 0 to 3: free of TMD.

- Values of 4 to 8: mild TMD.

- Values of 9 to 14: moderate TMD.

- Values of 15 to 23: severe TMD.

Then all patients were subjected to clinical examinations which include:

- TMJ pain on palpation: The tip of the index finger was placed over the lateral aspects of both joint area and slight pressure was applied, pain or tenderness was recorded in a static position or during opening and closing of the mouth. The same procedure was repeated to examine the posterior aspect of the TMJ via external audiatory meatus using the index and middle fingers, pain on movement or tenderness was recorded whenever palpation resulted in reflex or the patient reported a subject discomfort.

- Pain on movement of the mandible: The patient was asked to perform the following movements: opening and closing, right and left laterotrusion and protrusion, any pain felt by the subject during these movements.

- Masticatory muscles tenderness: By examining the tender points in the masticatory muscles according to Okesson JP (4) As follows:

- Masseter muscle: The deep part is palpated at its superior attachment the fingers are placed on the zygomatic arches just anterior to TMJ, and superficial part is palpated at its inferior attachment on the inferior borders of mandibular ramus.

- Temporalis muscle: Its superior part is palpated on the side of the head above and behind the ears and its anterior part is palpated above the zygomatic arch and anterior to TMJ and its insertion is palpated in the coronoid process intra orally along the anterior border of the 
ramus upward to the coronoid process.

- Lateral pterygoid muscle: Palpated by placing the index finger in the maxillary buccal vestibule, and the subject was instructed to move the mandible towards the side being palpated to gain better access through the shifting of the coronoid process. The palmar surface of the index finger is moved poseriorly, superiorly, and medially into the area of the infratemporal fossa, posterior to the maxillary tuberosity.

- Medial Pterygoid muscle: It is easily to examine intraorally with the mouth open. Placing the thump finger externally at the medial aspect of the angle of the mandible and the index finger intraorally in the lingual vestibule in the retromolar region ${ }^{(23)}$.

Joint noises: The presence of joint noises or clicking based on right and left TMJ inspection was also evaluated. This evaluation was carried out by placing the pointer fingers lightly upon the region corresponding to the lateral pole of the condyle, facing the external acoustic meatus, while the patient performed movements of mandibular opening and closing ${ }^{(24)}$.

Limitations in mouth opening: The vertical distance between the incisal surfaces of the upper and lower teeth was measured by a Vernier and recorded in millimeters $(\mathrm{mm})$. The minimum limit of normal oral opening was determined to be $34 \mathrm{~mm}^{(25)}$.

Headache Classification: Headaches were assessed using a validated structured questionnaire consisting of 26 questions. Questions largely mimic the structure of questionnaires used in very large epidemiological studies, and captures information on distinguishing headache features (frequency and duration of headacheepisodes, laterality, characteristics of pain, exacerbation with movement), and associated symptoms (aura, nausea, photophobia, phonophobia, autonomic symptoms). The questionnaire parallels the International Classification for Headache Disorders-II (26). On the basis of the responses and in consultation with neurologist, patients were categorized as having (TMD with no headaches, TMD with migraine, TMD with tension-type headache and TMD with headache in the temple area result from TMD which was not related to any other disorder. The ICDH-II tensiontype headache criteria were used as a framework to create a frequency/severity spectrum for classification of these temple headaches in this sample. The ICDHII Tension type headache (TTH) criteria are based on: A. headache frequency; B. headache duration;
C. headache quality; and D. absence or limited presence of migraine symptoms of nausea/ vomiting, photophobia and phonophobia ${ }^{(27)}$. While for migraine Diagnostic criteria:

a.At least two attacks fulfilling criteria $\mathrm{B}$ and $\mathrm{C}$

b.One or more of the following fully reversible aura symptoms: 1 . visual 2. sensory 3. speech and/or language 4. motor 5 . brainstem 6 . Retinal.

c.At least two of the following four characteristics: 1. At least one aura symptom spreads gradually over 5 minutes, and/or two or more symptoms occur in succession 2. Each individual aura symptom lasts 5-60 minutes 3. At least one aura symptom is unilateral 4. The aura is accompanied, or followed within 60 minutes, by headache.

d.Not better accounted for by another ICHD-II diagnosis, and transient ischaemic attack has been excluded ${ }^{(27)}$.

TTH criteria quality characteristics: bilateral location, pressing/tightening (non-pulsating) quality, or not aggravated by routine physical activity. Some of the patients within each of these types reported nausea, vomiting or phono- and photophobia but were not considered of migraine type due to meeting TTH criterion for headache quality. Inclusion of these patients was deemed appropriate as these symptoms are not exclusive to migraines and are exhibited by other chronic pain conditions ${ }^{(28)}$. Statistical analysis: Analysis of data was carried out using the available statistical package of SPSS-20 (Statistical Packages for Social Sciences-version 20) to compare between groups by means of $\chi 2$-tests and Microsoft Excel 2013 on windows $8, P$ values $<0.05$ were considered statistically significant.

\section{RESULTS}

The sample was consisted of (120) patients, the age ranged between (18-50) years with a mean age of (36) years, Gender distribution: It was found (72.5\%) female and $(27.5 \%)$ male (Table 1$)$. The sample was divided into four groups according to signs and symptoms of TMDs and headache questionaries', the first group consists of 30 (25\%) patients having TMD with migraine, the second group consists of $40(33.33 \%)$ patients having TMD with tension type headache, the third group consists of $25(20.83 \%)$ patients having TMD with headache from TMD, and the fourth group consists of $25(20.83 \%)$ patients having TMD without headache. The relation between the severity of TMD and gender was highly significant in female with p-value $(<0.01)$ as shown in (table 3$)$. 
The distribution of the severity of TMDs in relation to of TMDs in relation to primary headache was highly primary headache was highly significant in the patient significant in the patient with migraine and TTH with with migraine and TTH with p-value $(0.000)$ as shown $\mathrm{p}$-value $(<0.01)$ and in patients with headache from in (table 4). The distribution of Clinical examinations TMD with p-value $(<0.01)$ as shown in (table 5).

Table 1: Gender distribution.

\begin{tabular}{|c|c|c|c|}
\hline variables & Female & Male & \multicolumn{1}{|c}{} \\
\cline { 1 - 3 } $120(100 \%)$ & $87(72.5 \%)$ & $33(27.5 \%)$ & P value $<0.01$ \\
\hline
\end{tabular}

Table 2: TMD and primary headache distribution

\begin{tabular}{|c|c|c|c|c|}
\hline variables & $\begin{array}{c}\text { TMD with Migraine } \\
\text { No.-\% }\end{array}$ & $\begin{array}{c}\text { TMD with } \text { TTH } \\
\text { No.-\% }\end{array}$ & $\begin{array}{c}\text { TMD with Headache from TMD } \\
\text { No.-\% }\end{array}$ & $\begin{array}{c}\text { TMD without Headache } \\
\text { N0.-\% }\end{array}$ \\
\hline $120(100 \%)$ & $30(25 \%)$ & $40(33.33 \%)$ & $25(20.83 \%)$ & $25(20.83 \%)$ \\
\hline
\end{tabular}

Table 3: The severity of TMD in relation to gender

\begin{tabular}{|c|c|c|}
\hline $\begin{array}{c}\text { Variables } \\
(\boldsymbol{n}-\boldsymbol{0})\end{array}$ & $\begin{array}{c}\text { Male } \\
\text { 33(100\%) }\end{array}$ & $\begin{array}{c}\text { Female } \\
\mathbf{8 7}(\mathbf{1 0 0 \% )}\end{array}$ \\
\hline Mild & $7(21.21 \%)$ & $14(16.09 \%)$ \\
\hline Moderate & $26(87.87 \%)$ & $37(42.52 \%)$ \\
\hline Severe & $0(0 \%)$ & $36(41.37 \%)$ \\
\hline
\end{tabular}

p-value $<0.01$

Table 4:Distribution of The severity of Temporomandibular Disorders in relation to primary headache

\begin{tabular}{|c|c|c|c|c|}
\hline \multirow{2}{*}{ variables } & $\begin{array}{c}\text { TMD with } \\
\text { Migraine } \\
\text { N=30 }\end{array}$ & $\begin{array}{c}\text { TMD with } \\
\text { TTH } \\
\text { N=40 }\end{array}$ & $\begin{array}{c}\text { TMD with Head- } \\
\text { ache from } \text { TMD } \\
\text { N=25 }\end{array}$ & $\begin{array}{c}\text { TMD without } \\
\text { Headache } \\
\boldsymbol{N}=\mathbf{2 5}\end{array}$ \\
\hline Mild & $2(6.66 \%)$ & $1(2.5 \%)$ & $0(0 \%)$ & $18(72 \%)$ \\
\hline Moderate & $24(80 \%)$ & $23(57.5 \%)$ & $9(36 \%)$ & $7(28 \%)$ \\
\hline Severe & $4(13.33)$ & $16(40 \%)$ & $16(64 \%)$ & $0(0 \%)$ \\
\hline
\end{tabular}

p-value $<0.01$

Table 5: Distribution of Clinical examinations of TMDs in relation to primary headache

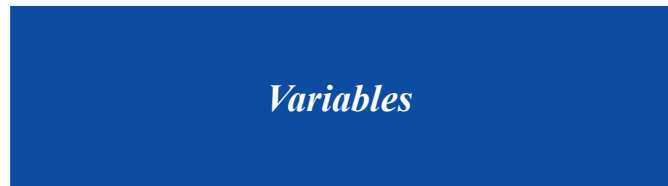

Presence of joint pain*
Presence of Masticatory muscles pain*
Presence of clicking**
Presence of Bruxism**
Presence of Limitation in mouth opening
$<34 \mathrm{~mm}^{* *}$

\section{DISCUSSION}

The relationship between headache and temporomandibular disorders has been documented in many clinical studies. Studies generally focused on the prevalence of temporomandibular disorders in headache patients, rather than on the prevalence of headache in temporomandibular disorders patients. A study carried out by Ballegaard et al. ${ }^{(29)}$ had investigated the coexistence of headache and temporomandibular disorders, examined 99 patients

\begin{tabular}{|c|c|c|}
\hline $\begin{array}{c}\text { TMD with } \\
\text { TTH }\end{array}$ & $\begin{array}{c}\text { Headache from } \\
\text { TMD }\end{array}$ & $\begin{array}{c}\text { TMD without } \\
\text { Headache }\end{array}$ \\
(Total No.=40) & $\begin{array}{c}\text { Total No.=25) } \\
\text { (Total No. }=25)\end{array}$ \\
\hline $36(90 \%)$ & $21(84 \%)$ & $11(44 \%)$ \\
\hline $33(88 \%)$ & $24(96 \%)$ & $12(48 \%)$ \\
\hline $31(77.5 \%)$ & $18(72 \%)$ & $14(56 \%)$ \\
\hline $30(75 \%)$ & $24(96 \%)$ & $4(16 \%)$ \\
\hline $29(72.50)$ & $16(64 \%)$ & $4(16 \%)$ \\
\hline
\end{tabular}

with headache, temporomandibular disorders was reported in female more than male as shown in present study. Another study documented by Schokker et al. (30) had been showed that approximately half of the subjects with recurrent headache had reported pain during mouth opening as wide as possible, whereas a high proportion of headache patients had reported tension of the masseter and temporal muscles. Graff-Radford (31) studied the relationship between temporomandibular disorders and headache, and 
vice versa, and demonstrated that possible causes of headache included temporomandibular joint inflammation, temporomandibular joint arthropathy and joint disk displacement. In patients with TTH increased headache has been found following jaw movements or clenching of the teeth. This kind of close temporal relationship between headache and a causative disorder is one of the principle criteria for secondary headache diagnoses ${ }^{(31)}$.

In the present study primary headache (migraine and TTH) is a prevalent condition in TMD patients

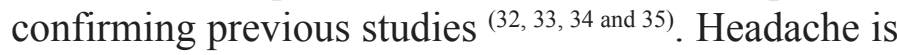
a possible cause of chronic pain or TMD. By using anamnestic index in this study, TMD Severity score in Female was higher than for male, this finding confirming several previous studies that have shown the existence of an epidemiological difference between males and females. Among women these rates are higher probably due to the influence of behavioral, hormonal, anatomic and psychosocial factors $(36,37$, and 38$)$.

In the present study, the clinical signs and symptoms of TMD, such as tenderness in masticatory muscles, TMJ pain, clicking, limitation in mouth opening, and Bruxism were also statically more severe in headache patients, than those with TMD without Headache ones this finding approving with other studies that also found a higher prevalence of masticatory muscle tenderness and TMJ pain in headache patients $(39,40$, and 41 ).

Clinical and epidemiological studies have demonstrated a connection between headache and TMDs, suggesting that individuals with headache and those with TMDs, often show similar signs and symptoms in their clinical presentations. Patients with sleep bruxism (SB) may not always show significant pain in the TMDs. SB may produce subclinical nociceptive signaling from the stomatagnathic structures to the trigeminal nucleus increasing central sensitization. Recognition, Evaluation and effective management of the SB patient has the potential to increase headache treatment efficacy and potentially reduce the need for pharmacotherapy. Christensen ${ }^{(48)}$ documented that muscle pain was demonstrated in patients who voluntarily clenched for 20-30 seconds. Kydd and Daly ${ }^{(49)}$ reported that nocturnal clenching events can last as long as 20-40 seconds. These sustained isometric contractions observed in sleep bruxism could lead to tissue injury and subsequent nociceptive signaling from both the muscular and joint components of the temporomandibular joint complex. Previous researches have also reported mechanisms for nociceptive referral from the temporomandibular joints (TMJs) to the cranial structures ${ }^{(50,51)}$.

The present study and several studies have reported the overlapping signs and symptoms of temporomandibular disorders (TMD) in tension-type headache (TTH) patients and TTH in TMD patients, respectively $(42,43,44,45$, and 46$)$.

Migraine headache and TMD are both characterized by pain in the head and/or face and both conditions are more common in women as shown in present study, particularly in their child-bearing years. Migraine headache and TMD showed a high prevalence in women in comparing to men. Women in present study represented $72.5 \%$ of all TMD patients and a majority of headache patients partly because of their increased likelihood of seeking care. Due to the close anatomical relationship of the muscles of mastication and TMJ to the head and due to the frequency of referred pain, it is sensible to theorize that there is a significant percentage of patients with headache actually have TMD as the major source of their pain ${ }^{(47)}$.

\section{CONCLUSIONS}

High prevalence of TMD, especially in females and also females represent a majority of headache patients partly because of their increased likelihood of seeking care and due to the influence of behavioral, hormonal, anatomic and psychosocial factors. Clinical pain characteristics and masticatory muscle tenderness of TMD is greater in patients with primary headache and the provocation of the masticatory system more likely to result in their headache. Clinically, the presence of joint pain masticatory muscles pain, clicking, bruxism and limitation in mouth opening are greater in patients with TTH and Migraine.

\section{REFERENCES}

1. Okeson JP. Signs and symptoms of temporomandibular disorders. In: (ed.) Okeson JP. Management of temporomandibular disorders and occlusion. 6th ed. St Louis: Mosby Elsevier.2008:164-215

2. Okeson JP, de Leeuw R. Differential diagnosis of temporomandibular disorders and other orofacial pain disorders. Dent Clin North Am. 2011;55:105-20.

3. Scully, Crispian (2008). Oral and maxillofacial medicine: the basis of diagnosis and treatment (2nd ed.). Edinburgh: Churchill Livingstone. pp.8,14,30,31,33,101,104,106,291

4. OkessonJP.Etiology and identification of functional disturbances in masticatory system. In management of tempromandibular disorders and occlusion(5th ed.) mosby publication.2003;147-364.

5. Cawson RA, Odell EW, Porter S (2002).Cawsonś essentials of oral pathology and oral medicine (7th ed.). Edinburgh: Churchill Livingstone. ISBN 0-443-07106-3. 
6. Wright, Edward F. (2010). Manual of temporomandibular disorders (2nd ed.). Ames, IA: Wiley-Blackwell. ISBN 9780-8138-1324-0

7. Wassell R, Naru A, Steele J, Nohl F (2008). Applied occlusion. London: Quintessence. pp. 73-84. ISBN 978-185097-098-9

8. Rossetti LM, Pereira de Araujo Cdos R, Rossetti PH, Conti PC. Association between rhythmic masticatory muscle activity during sleep and masticatory myofascial pain: a polysomnographic study. J Orofac Pain. 2008 Summer. 22(3):190-200.

9. ICHD-2: 2nd Edition International Classification of Headache Disorders “. International Headache Society. Retrieved 7 May 2013.

10. Anderson, GC; Gonzalez, YM; Ohrbach, R; Truelove, EL; Sommers, E; Look, JO; Schiffman, EL (Winter 2010). Ann Rheum Dis 1981;40:366-9.

11. L. LeResche, S. F. Dworkin, L. Wilson, and K. J. Ehrlich, "Effect of temporomandibular disorder pain duration on facial expressions and verbal report of pain," Pain, vol. 51, no. 3, pp. 289-295, 1992.

12. V. Ballegaard, P. Thede-Schmidt-Hansen, P. Svensson, and R. Jensen, "Are headache and temporomandibular disorders related? A blinded study," Cephalalgia, vol. 28, no. 8, pp. 832- 841, 2008.

13. Schiffman E, Halet D, Baker C, Lindgren B. Diagnostic criteria for screening headache patients for temporomandibular disorders. Headache. 1995;35:121-4.

14. Williamson EH. Interrelationship of internal derangements of the temporomandibular joint, headache, vertigo, and tinnitus: a survey of 25 patients. Cranio. 1990;8:301-6.

15. Quayle AA, Gray RJ, Metcalfe RJ, Guthrie E, Wastell D. Soft occlusal splint therapy in the treatment of migraine and other headaches. J Dent. 1990;18:123-9.

16. Steele JG, Lamey PJ, Sharkey SW, Smith GMR. Occlusal abnormalities, pericranial muscle and joint tenderness and tooth wear in a group of migraine patients. J Oral Rehabil. 1991;18:453-8.

17. Schokker RP, Hansson TL, Ansik BJJ. Craniomandibular disorders in patients with different types of headache. J Craniomand Disord Facial Oral Pain. 1990;4:47-51.

18. Schellhas KP, Wilkes CH, Baker CC. Facial pain, headache, and temporomandibular joint inflammation. Headache. 1989;29:228-31.

19. Franco AL, Gonçalves DAG, Castanharo SM, Speciali JG, Bigal ME, Camparis CM. Migraine is the most prevalent primary headache in Individuals with temporomandibular disorders. J Orofac Pain. 2010;24:287-92.

20. Headache Classification Subcommittee of the International Headache Society. The International Classification of Headache Disorders. Cephalalgia. 2004;24(suppl 1):1:160.

21. McNeill C, Management of temporomandibular disorders: concepts and controversies1997 May;77(5):510-22.

22. Helkimo M. Studies on function and dysfunction of the masticatory system. 3. Analyses of anamnestic and clinical recordings of dysfunction with the aid of indices. Sven Tandlak Tidskr. 1974; 67(3):165-81.

23. Travell and Simons: Myofacial pain and dysfunction the trigger point manual. Vol 1 upper half of the body, 2nd edition, 1999.

24. Austin DG, Pertes RA. Examination of the dysfunction patients. In: Pertes RA, Gross SG, editors. Clinical management of temporomandibular disorders and oroafacial pain. Chicago: Quintessence; 1995. p. 123-60.

25. Gallagher C, Gallagher V, WheltonH,Crownin M. The normal range of mouth opening,2004.

26. Lipton RB, Stewart WF, Diamond S, et al. Prevalence and burden of migraine in the United States: data from the American Migraine Study II. Headache. 2001;41:646-657

27. International Headache Society. The International Classification of Headache Disorders. Cephalalgia. 2004; $24 \quad 1: 37-43$.

28. Watson NF, Buchwald D, Goldberg J, Noonan C, Ellenbogen RG. Neurologic signs and symptoms in fibromyalgia. Arthritis Rheum. 2009 Sep; 60(9):2839-44. [PubMed: 19714636]

29. BALLEGAARD V, THEDE-SCHMIDT-HANSEN $P$, SVENSSON P, JENSEN R. Are headache and temporomandibular disorders related? A blinded study. Cephalalgia 2008; 28: 832-841.

30. SCHOKKER RP, HANSSON TL, ANSINK BJ. Craniomandibular disorders in patients with different types of headache. J Craniomandib Disord 1990; 4: 47-51.

31. GRAFF-RADFORD SB. Temporomandibular disorders and headache. Dent Clin North Am 2007; 51: 129-144.

32. Franco AL, Gonçalves DA, Castanharo SM, Speciali JG, Bigal ME, Camparis CM. Migraine is the most prevalent primary headache in individuals with temporomandibular disorders. J Orofac Pain. 2010;24(3):287-92.

33. Gonçalves DA, Bigal ME, Jales LC, Camparis CM, Speciali JG: Headache and symptoms of temporomandibular disorder: an epidemiological study. Headache. 2010;50(2):231-41. doi:10.1111/j.1526-4610.2009.01511.

34. Ballegaard V, Thede-Schmidt-Hansen P, Svensson P, Jensen $\mathrm{R}$ : Are headache and temporomandibular disorders related? a blinded study. Cephalalgia. 2008;28(8):832-41. doi:10.11 11/j.1468-2982.2008.01597.

35. Stuginski-Barbosa J, Macedo HR, Bigal ME, Speciali JG. Signs of temporomandibular disorders in migraine patients: a prospective, controlled study. Clin J Pain. 2010;26(5):41821. doi:10.1097/AJP.0b013e3181d1069.

36. Nilsson IM, Drangsholt $M$, List $T$. Impact of temporomandibular disorder pain in adolescents: differences by age and gender. J Orofac Pain 2009;23:115122 .

37. Manfredini D, Piccotti F, Ferronato G, Guarda-Nardini L . Age peaks of different RDC/TMD diagnoses in a patient population. J Dent 2010;38:392-399.

38. Slade GD, Bair E, Greenspan JD, Dubner R, Fillingim RB, Diatchenko L, Maixner W, Knott C, Ohrbach R. Sings and symptoms of first-onset TMD and sociodemographic predictors of its development: the OPPERA prospective cohort study. J Pain 2013;14:T20-T32.

39. Fragoso YD, Alves HHC, Garcia SO, Finkelsztejn A. Prevalence of parafunctional habits and temporomandibular dysfunction symptoms in patients attending a tertiary headache clinic. Arq Neuropsiquiatr. 2010;68:377-80.

40. Graff-Radford SB.Temporomandibular disorders and headache. Dent Clin North Am. 2007;51:129-44.

41. Gonçalves DA, Bigal ME, Jales LC, Camparis CM, Speciali JG. Headache and symptoms of temporomandibular disorder: an epidemiological study. Headache. 2010;50:23141.

42. Carra MC, Huynh N, Morton P, Rompré PH, Papadakis A, Remise C,Université de Montréal, QC, Canada, et al: Prevalence and risk factors of sleep bruxism and wake-time 
tooth clenching in a 7 - to 17 -yr-old population. Eur J Oral Sci 2011, 119(5):386-394 44.

43. HuynhN, Kato T, RomprePH, etal. Sleepbruxismisassociated to micro-arousals and an increase in cardiac sympathetic activity. Journal of sleep research2006;15(3):339-46 .

44. Glaros AG, Urban D, Locke J. Headache and temporomandibular disorders: evidence for diagnostic and behavioural overlap. Cephalalgia. 2007;27:542-9.

45. Poveda-Roda R, Bagán JV, Jiménez-Soriano Y, FonsFont A. Retrospective study of a series of 850 patients with temporomandibular dysfunction (TMD). Clinical and radiological findings. Med Oral Patol Oral Cir Bucal.2009; 14:e628-34.

46. Crystal SC, Robbins MS. Epidemiology of tension-type headache. Curr Pain Headache Rep 2010;14:449-454.
47. V. Demarin, V. Bašić Kes: Temporomandibular disorders and migraine headache. Rad 507. Medical Sciences, 34(2010):111-117.

48. Christensen LV. Facial pain from experimental tooth clenching. Tandlaegebladet 1970;74(2):175-82.

49. Kydd WL, Daly C. Duration of nocturnal tooth contacts during bruxing. J Prosthet Dent 1985;53(5):717-21.

50. Cairns BE, Sessle BJ, Hu JW. Evidence that excitatory amino acid receptors within the temporomandibular joint region are involved in the reflex activation of the jaw muscles. The Journal of neuroscience : the official journal of the Society for Neuroscience 1998;18(19):8056-64.

51. Thalakoti S, Patil VV, Damodaram S, et al. Neuron-glia signaling in trigeminal ganglion: implications for migraine pathology. Headache 2007;47(7):1008-23; discussion 24-5. 